\title{
Nearly scale-invariant power spectrum and quantum cosmological perturbations in the gravity's rainbow scenario
}

\author{
Sai Wang ${ }^{1, a}$, Zhe Chang ${ }^{1,2}$ \\ ${ }^{1}$ State Key Laboratory of Theoretical Physics, Institute of Theoretical Physics, Chinese Academy of Sciences, Beijing 100049, China \\ ${ }^{2}$ Institute of High Energy Physics, Chinese Academy of Sciences, Beijing 100049, China
}

Received: 22 December 2014 / Accepted: 13 May 2015 / Published online: 11 June 2015

(C) The Author(s) 2015. This article is published with open access at Springerlink.com

\begin{abstract}
We propose the gravity's rainbow scenario as a possible alternative of the inflation paradigm to account for the flatness and horizon problems. We focus on studying the cosmological scalar perturbations which are seeded by the quantum fluctuations in the very early universe. The scalar power spectrum is expected to be nearly scale-invariant. We estimate the rainbow index $\lambda$ and energy scale $M$ in the gravity's rainbow scenario by analyzing the Planck temperature and WMAP polarization datasets. The constraints on them are given by $\lambda=2.933 \pm 0.012$ and $\ln \left(10^{5} M / M_{p}\right)=$ $-0.401_{-0.451}^{+0.457}$ at the $68 \%$ confidence level.
\end{abstract}

\section{Introduction}

The inflation model [1-3] has been the leading paradigm for the very early universe in the last three decades. In the inflation paradigm, the scale factor $a(t)$ of the universe has undergone a stage of exponential expansion in a very short time. This leads to a universe flat enough to account for the flatness problem, since $\left|\Omega_{K}\right| \propto a^{-2}$. On the other hand, this reveals that the cosmological scales observed today were deep inside the Hubble scale, which accounts for the horizon problem. Moreover, the cosmological scalar perturbations can be seeded by the primordial quantum fluctuations which are stretched outside of the horizon (see [4] for reviews). The scalar power spectrum is predicted to be adiabatic, Gaussian, and nearly scale-invariant. This is well consistent with the present astronomical observations on the anisotropy of cosmic microwave background and the formation of large-scale structures. Although the inflation fits the observational data well, it still suffers several significant issues, such as the finetuning slow-roll potential [5], the initial conditions [6,7], and the trans-Planckian problem [8], etc. In addition, one requires an inflaton field to drive the exponential expansion of the very early universe. However, the astronomical observations

\footnotetext{
a e-mail:wangsai@itp.ac.cn
}

have not yet discovered such a fundamental scalar field until recently.

It is interesting to study possible alternatives for the inflation paradigm. In an alternative scenario, actually, one just needs to require that the observed universe were inside the particle horizon in the very early universe to account for the problems of big-bang cosmology. In this paper, we propose that the gravity's rainbow scenario shall meet this requirement. The gravity's rainbow scenario [9] has arisen from the phenomenological studies of the quantum gravity which should play a significant role in the very early universe. Recently, it has been utilized to study the very early universe [10-20]. The spacetime metric felt by a free particle would be dependent on the energy (or momentum, equivalently) of the particle in the gravity's rainbow scenario. Thus the dispersion relation can be significantly modified for a ultra-relativistic particle. This leads to an effective speed of light. The varying speed of light cosmology has been proposed [21-23], and the observable universe was assumed to be only a part of the causal area if the effective speed of light is large enough in the very early universe. Thus, the gravity's rainbow scenario shall have potential to resolve the flatness and horizon problems.

In the gravity's rainbow scenario, the evolution of the very early universe would be driven by the thermally fluid substance instead of a fundamental scalar field. We will study the thermodynamics of the system of ultra-relativistic particles with the modified dispersion relation. Then the background evolution of the universe is determined by the modified Friedmann equation. The solution of the Friedmann equation will be showed to resolve the flatness and horizon problems. We shall focus on studying the cosmological linear perturbations and their quantization in this paper. The issue of gauge choices will be studied in detail, and then the perturbed Einstein's field equations will be calculated in the longitudinal gauge. We will construct the comoving curvature perturbation which is gauge-invariant and conserved outside 
the Hubble horizon. In this model, the quantum fluctuations are expected to dominate above the rainbow energy scale. Furthermore, we will set constraints on the parameters of the gravity's rainbow effects by a joint analysis of the Planck temperature [24] and WMAP polarization [25] datasets.

The rest of the paper is arranged as follows. In Sect. 2, we study the evolution of the background spacetime in the gravity's rainbow scenario. In Sect. 3, the equations of motion are derived for the scalar perturbations. We quantize the scalar perturbations in the longitudinal gauge in Sect. 4. In Sect. 5, we calculate the power spectrum of the primordial scalar perturbations and then set constraints on the parameters of the gravity's rainbow effects. The conclusions and discussions are given in Sect. 6.

\section{Evolution of background}

The gravity's rainbow scenario was originally studied by Magueijo and Smolin [9]. The spacetime metric felt by a free particle depends on the energy or momentum of the particle. In the study of cosmology, we are interested in the spatially flat Friedmann-Robertson-Walker (FRW) metric which is homogeneous and isotropic. In this paper, we will study the evolution of the very early universe with the modified FRW metric of the form [26]

$\mathrm{d} \tau^{2}=c^{2}(p) \mathrm{d} t^{2}-a^{2}(t) \delta_{i j} \mathrm{~d} x^{i} \mathrm{~d} x^{j}$,

where $a(t)$ is the scale factor of the universe, the rainbow function $c(p)$ is explicitly parameterized as a power-law form, namely,

$c(p)=1+(p / M)^{\lambda}$.

Here $M$ is an energy scale related to the quantum gravity, and $\lambda$ is called the rainbow index which is positive. The rainbow function $c(p)$ takes the $\operatorname{limit}_{\lim _{p / M \rightarrow 0}} c(p)=1$. In the tangent space, the metric (1) would lead to the modified dispersion relation for a free ultra-relativistic particle. This could be given as

$E=c(p) p=p+M^{-\lambda} p^{\lambda+1}$,

where we have neglected the mass term for the particle, since the particle's mass is tiny compared to the ultra-relativistic energy.

In an early enough era of the universe, the particle could have an extremely high energy scale, i.e., $p \gg M$. Then the rainbow function becomes

$c(p) \simeq(p / M)^{\lambda}$.

Thus the second term at the right hand side in (3) will dominate, namely,
$E \simeq M^{-\lambda} p^{\lambda+1}$.

Consider a system of such ultra-relativistic particles in thermal equilibrium. It should meet the Maxwell-Boltzmann distribution. We can obtain the energy density $\rho(T)$ of the system with the temperature $T$, namely,

$$
\begin{aligned}
\rho(T) & =\int_{0}^{\infty} 4 \pi p^{2} E e^{-E / T} \mathrm{~d} p \\
& =\frac{4 \pi}{\lambda+1} M^{\frac{3 \lambda}{\lambda+1}} \int_{0}^{\infty} E^{\frac{3}{\lambda+1}} e^{-E / T} \mathrm{~d} E \\
& =\sigma(\lambda, M) T^{\frac{\lambda+4}{\lambda+1}} \propto T^{\frac{\lambda+4}{\lambda+1}},
\end{aligned}
$$

where the constant coefficient $\sigma(\lambda, M)$ is given as

$\sigma(\lambda, M)=\frac{4 \pi}{\lambda+1} \Gamma\left(\frac{\lambda+4}{\lambda+1}\right) M^{\frac{3 \lambda}{\lambda+1}}$.

In the second equality of (6), we used the relation (5). The pressure $P(T)$ of the system could be obtained by resolving the ordinary differential equation

$T \frac{\mathrm{d} P}{\mathrm{~d} T}-P=\rho$.

Thus, it is given by

$P(T)=\frac{\lambda+1}{3} \sigma T^{\frac{\lambda+4}{\lambda+1}}=\omega \rho(T)$,

where we neglected an integral constant, and the state parameter is given by

$\omega=\frac{\lambda+1}{3}$.

The relation (9) is just the so-called equation of state. In addition, the speed of sound could be obtained as $c_{s}^{2}:=\frac{\partial P}{\partial \rho}=$ $\frac{\lambda+1}{3}$, which is also a constant. When $\lambda=0$, the above results on $\rho, P, w$, and $c_{s}$ would return back to the conventional form for the massless particles (such as the photons) in special relativity.

The conservation of energy-momentum tensor gives the equation of continuity for the thermodynamic system. For a system of an ideal fluid, the energy-momentum tensor is given by

$T_{v}^{\mu}=(\rho+P) u^{\mu} u_{v}-P \delta_{v}^{\mu}$,

where $u^{\mu} u_{\mu}=1$. Its conservation implies the equation $T_{0 ; \mu}^{\mu}=0$. Implicitly, this equation can be written as the equation of continuity,

$\frac{\mathrm{d} \rho}{\mathrm{d} t}+3 H(\rho+P)=0$, 
where the Hubble parameter $H=\dot{a} / a$ and $\dot{a}=\mathrm{d} a / \mathrm{d} t$. By combining (9) with (12), we obtain

$\rho=\rho_{e}\left(\frac{a}{a_{e}}\right)^{-3(1+\omega)} \propto a^{-(\lambda+4)}$.

Hereafter the subscript " $e$ " denotes physical quantities at the moment when the gravity's rainbow effect no longer dominates, i.e., $p_{e} \simeq M$. By comparing (13)-(6), we obtain a useful relation, i.e.,

$a \propto T^{-\frac{1}{\lambda+1}}$,

or, equivalently, $a=a_{e}\left(T / T_{e}\right)^{-\frac{1}{\lambda+1}}=a_{e}(T / M)^{-\frac{1}{\lambda+1}}$. Here $a_{e}$ can be roughly estimated at the temperature $T_{e}=M$ by the $\Lambda$ CDM model.

The evolution of the scale factor $a(t)$ is determined by the Friedmann equation, which is deduced from the Einstein field equation [27]. In this paper, we assume the modified Einstein's equation as follows:

$G_{v}^{\mu}=\frac{1}{c^{4}} T_{v}^{\mu}$,

where $G_{\mu \nu}$ is the Einstein tensor. In this paper, we set $M_{p}^{-2}=$ $8 \pi G=1$. The rainbow function $c$ appears as the effective speed of light in the above equation. Then the 00-component of Einstein's equation gives the Friedmann equation,

$H^{2}=\frac{1}{3 c^{2}} \rho$.

In the Friedmann equation, one takes the ultra-relativistic particles as an ensemble rather than picking out a specific particle randomly [26]. One should take into account the average effects of the ensemble on the evolution of the very early universe. By using (4) and (5), we obtain $c(E)=(E / M)^{\frac{\lambda}{\lambda+1}}$. As an ensemble, the thermodynamic system in thermal equilibrium has a typical energy scale, namely, the temperature $T$ takes a statistical mean value. Thus, one could take $T$ as the energy appearing in the gravity's rainbow metric, namely,

$c \equiv c(T)=(T / M)^{\frac{\lambda}{\lambda+1}}$.

Then we could resolve the Friedmann equation (16), and the solution is

$a(t) \propto t^{\frac{2}{4-\lambda}}$,

where we used (14) and (17). We set $\lambda<4$ to obtain an expanding universe, while $\lambda>4$ is related to a contracting universe. If $\lambda=4$, the exponent $2 /(4-\lambda)$ would be divergent. This case is not well defined.

The flatness and horizon problems can be demonstrated as follows. The spatial curvature term $\left|\Omega_{k}\right|=\frac{c^{2}}{a^{2} H^{2}}$ is pro- portional to $T^{\frac{3 \lambda-2}{\lambda+1}}$. With the decrease of temperature in the expanding universe, $\left|\Omega_{k}\right|$ should also decrease across more than 24 orders of magnitude to resolve the flatness problem. Then we require $\lambda>2 / 3$ and a high energy scale $T_{i} \gg T_{e}$. Hereafter the subscript " $i$ " denotes the start time of the rainbow universe. On the other hand, the particle horizon $\mathrm{d}_{H}=\int_{t_{i}}^{t_{e}} \frac{c \mathrm{~d} t}{a}=\int_{a_{i}}^{a_{e}} \frac{c \mathrm{~d} a}{a^{2} H}$ is proportional to $T^{\frac{3 \lambda-2}{2(\lambda+1)}}$. Similarly to resolving the flatness issue, it also requires $\lambda>2 / 3$ to resolve the horizon problem. If $\lambda>4$, however, the temperature would increase with the increase of the time based on (14) and (18). This makes even worse the flatness and horizon problems. Thus, the above discussions show that the rainbow index $\lambda$ should satisfy the condition $2 / 3<\lambda<4$.

\section{Scalar perturbations}

In the following, we shall focus on studying the cosmological linear perturbations and their quantization, while disregarding the statistically thermal fluctuations ${ }^{1}$. The rainbow function $c(T)$ could be formally viewed as a smooth background function of the temperature. Consider the scalar perturbations. The perturbed rainbow metric takes the form

$$
\begin{aligned}
\mathrm{d} \tau^{2}= & a^{2}\left[(1+2 \phi) c^{2} \mathrm{~d} \eta^{2}+2 c B_{, i} \mathrm{~d} x^{i} \mathrm{~d} \eta\right. \\
& \left.-\left((1-2 \psi) \delta_{i j}-2 E_{, i j}\right) \mathrm{d} x^{i} \mathrm{~d} x^{j}\right],
\end{aligned}
$$

where we have used the conformal time $\mathrm{d} \eta=\mathrm{d} t / a$. We consider the coordinate transformation $x^{\mu} \rightarrow \tilde{x}^{\mu}=x^{\mu}+\zeta^{\mu}$. Here $\zeta^{\mu}=\left(\zeta^{0}, \zeta^{i}\right)$ denotes the infinitesimal functions of the spacetime coordinates, and $\zeta^{i}=\zeta_{\perp}^{i}+\varsigma^{, i}$ where $\zeta_{\perp, i}^{i}=0$ and $\zeta$ denotes a scalar function. The Lie derivative of the metric perturbations $\delta g_{\mu \nu}(x)=g_{\mu \nu}(x)-\bar{g}_{\mu \nu}(x)$ gives the gauge transformation law, i.e., $\delta g_{\mu \nu} \rightarrow \delta \tilde{g}_{\mu \nu}=$ $\delta g_{\mu \nu}-\bar{g}_{\mu \nu, \sigma} \zeta^{\sigma}-\bar{g}_{\sigma \nu} \zeta_{, \mu}^{\sigma}-\bar{g}_{\mu \sigma} \zeta_{, \nu}^{\sigma}$, where $\bar{g}_{\mu \nu}$ denotes the unperturbed background metric. Thus, we can obtain

$$
\begin{aligned}
\phi & \rightarrow \tilde{\phi}=\phi-\frac{1}{a c}\left(a c \zeta^{0}\right)^{\prime}, \\
\psi & \rightarrow \tilde{\psi}=\psi+\frac{a^{\prime}}{a} \zeta^{0}, \\
B & \rightarrow \tilde{B}=B+\frac{1}{c} \varsigma^{\prime}-c \zeta^{0}, \\
E & \rightarrow \tilde{E}=E+\varsigma .
\end{aligned}
$$

Hereafter, the primes denotes the derivative with respect to the conformal time $\eta$. The Bardeen potentials are the simplest gauge-invariant linear combinations of the above scalar perturbations. They are given by

\footnotetext{
1 The power spectrum for the statistical thermal fluctuations is proportional to $T^{\frac{3(2--3 \lambda)}{2(1+\lambda)}}$ in the gravity's rainbow scenario. When $\lambda \simeq 3$, it will decrease rapidly with the increase of the background temperature. Thus, the thermal fluctuations would be suppressed in this scenario.
} 
$\Phi=\phi-\frac{1}{a c}\left[a\left(B-\frac{1}{c} E^{\prime}\right)\right]^{\prime}$,

$\Psi=\psi+\frac{a^{\prime}}{a c}\left(B-\frac{1}{c} E^{\prime}\right)$.

In the longitudinal gauge, we choose the system of coordinates with $B=E=0$. Thus, the perturbed rainbow metric (19) can be rewritten as

$\mathrm{d} \tau^{2}=a^{2}\left[(1+2 \Phi) c^{2} \mathrm{~d} \eta^{2}-(1-2 \Psi) \delta_{i j} \mathrm{~d} x^{i} \mathrm{~d} x^{j}\right]$

If the anisotropic stresses are not considered, we can obtain the relation $\Psi=\Phi$, as will be demonstrated later.

To derive the equations for the linear cosmological perturbations, we can linearize the Einstein field equations

$\delta G_{v}^{\mu}=\frac{1}{c^{4}} \delta T_{v}^{\mu}$

where $\delta G_{v}^{\mu}$ and $\delta T_{v}^{\mu}$ denote the gauge-invariant perturbations. In general, the perturbed energy-momentum tensor is given by

$\delta T_{0}^{0}=\delta \rho, \quad \delta T_{i}^{0}=\rho(1+\omega) v_{, i}, \quad \delta T_{j}^{i}=-c_{s}^{2} \delta \rho \delta_{j}^{i}$,

where we have neglected the anisotropic stress. Only the adiabatic perturbations are considered in this paper. Thus, the spatial components of the Einstein field equations can be explicitly given by

$$
\begin{gathered}
{\left[\Psi^{\prime \prime}+\mathscr{H}(2 \Psi+\Phi)^{\prime}+\left(2 \mathscr{H}^{\prime}+\mathscr{H}^{2}\right) \Phi+\frac{c^{2}}{2} \Delta(\Phi-\Psi)\right] \delta_{j}^{i}} \\
-\mathscr{H} \frac{\mathrm{d} \ln c}{\mathrm{~d} \ln a}\left(\Psi^{\prime}+2 \mathscr{H} \Phi\right) \delta_{j}^{i}-\frac{c^{2}}{2}(\Phi-\Psi)_{, j}^{, i}=-\frac{1}{2 c^{2}} a^{2} \delta T_{j}^{i} .
\end{gathered}
$$

Here $\mathscr{H}=a^{\prime} / a$ denotes the comoving Hubble parameter. For $i \neq j$, we have $\delta T_{j}^{i}=0$, and then (29) is reduced to $(\Phi-\Psi)_{, i j}=0$. The only solution is $\Psi=\Phi$, which is similar to the result in the standard model [27]. By considering this result, we obtain the following equations for the scalar perturbations:

$$
\begin{aligned}
& c^{2} \Delta \Phi-3 \mathscr{H}\left(\Phi^{\prime}+\mathscr{H} \Phi\right)=\frac{1}{2 c^{2}} a^{2} \delta \rho, \\
& \left(\Phi^{\prime}+\mathscr{H} \Phi\right)_{, i}=\frac{1}{2 c^{3}} a^{2} \rho(1+\omega) v_{, i}, \\
& {\left[\Phi^{\prime \prime}+3 \mathscr{H} \Phi^{\prime}+\left(2 \mathscr{H}^{\prime}+\mathscr{H}^{2}\right) \Phi\right]-\mathscr{H} \frac{\mathrm{d} \ln c}{\mathrm{~d} \ln a}\left(\Phi^{\prime}+2 \mathscr{H} \Phi\right)} \\
& =\frac{1}{2 c^{2}} a^{2} c_{s}^{2} \delta \rho .
\end{aligned}
$$

By combining (30) and (32), we obtain an equation for the gravitational potential $\Phi$, namely,

$$
\begin{aligned}
\Phi^{\prime \prime} & +3 \mathscr{H}\left(1+c_{s}^{2}-\frac{1}{3} \frac{\mathrm{d} \ln c}{\mathrm{~d} \ln a}\right) \Phi^{\prime}-c_{s}^{2} c^{2} \Delta \Phi \\
& +\left[2 \mathscr{H}^{\prime}+\mathscr{H}^{2}\left(1+3 c_{s}^{2}-2 \frac{\mathrm{d} \ln c}{\mathrm{~d} \ln a}\right)\right] \Phi=0 .
\end{aligned}
$$

Before resolving (33), we shall discuss the comoving curvature perturbation. This is a gauge-invariant quantity which is conserved outside the Hubble horizon. In general, it is defined by

$\mathscr{R}=-\Phi-\frac{1}{c} \mathscr{H} v$

Outside the Hubble horizon, one can disregard the terms proportional to $\triangle \Phi$. By combining (30) and (31), thus, one gets the equation $c \delta \rho+3 \mathscr{H}(\rho+P) v=0$. Therefore, the comoving curvature perturbation can be rewritten as

$\mathscr{R}=-\Phi-\frac{\delta \rho}{3(\rho+P)}$

Its derivative with respect to time is given by

$\mathscr{R}^{\prime}=\frac{\rho^{\prime} \delta P-P^{\prime} \delta \rho}{3(\rho+P)^{2}}$,

where we have used the equations for the energy-momentum conservation, i.e., $\rho^{\prime}+3 \mathscr{H}(\rho+P)=0$ and $\delta \rho^{\prime}+3 \mathscr{H}(\delta \rho+$ $\delta P)-3(\rho+P) \Phi^{\prime}=0$. Noting $P=\omega \rho$ and $\omega$ is a constant, the right-handed term must vanish in (36). Thus, $\mathscr{R}$ is conserved outside the Hubble horizon.

\section{Quantizing perturbations}

Equation (33) for the gravitational potential $\Phi$ can be reduced into a simpler form. In this paper, we just consider the case of $\lambda>2$, for which the reason will become clear later. By noting $a \propto(-\eta)^{\frac{2}{2-\lambda}}, c_{s}^{2}=\frac{1+\lambda}{3}$, and $c \propto a^{-\lambda}$, we obtain the equation

$\Phi^{\prime \prime}+2 q \eta^{-1} \Phi^{\prime}+\left(-m \eta^{-\ell} \Delta+\bar{n} \eta^{-2}\right) \Phi=0$,

where $q=\frac{2(2+\lambda)}{2-\lambda}, \ell=\frac{4 \lambda}{2-\lambda}, \bar{m}=\frac{\lambda+1}{3} \eta_{e}^{\ell}$, and $\bar{n}=\frac{16 \lambda}{(2-\lambda)^{2}}$. Here we have chosen the end moment of the gravity's rainbow effects as the original point of time. Hereafter, the subscript "e" denotes the quantity at the moment when the gravity's rainbow effects no longer dominate. One can introduce a new variable to eliminate the term proportional to $\Phi^{\prime}$. The new variable is given by $u=\left(-\eta / \eta_{e}\right)^{q} \Phi$. Then Eq. (37) becomes

$u^{\prime \prime}-\left(m \bar{\eta}^{-\ell} \Delta+n \bar{\eta}^{-2}\right) u=0$

where $\bar{\eta}=-\eta / \eta_{e}, m=\frac{\lambda+1}{3} \eta_{e}^{2}$, and $n=q(q-1)-\bar{n}=$ $2\left(4+3 \lambda^{2}\right) /(2-\lambda)^{2}$. Hereafter the prime denotes the derivatives with respect to $\bar{\eta}$. To quantize the new perturbation $u$, 
Eq. (38) can be put in correspondence to the action of the form

$S=\int \mathscr{L} \mathrm{d} \bar{\eta} \mathrm{d} x^{3}=\int \frac{1}{2}\left(u^{\prime 2}+m \bar{\eta}^{-\ell} u \Delta u+n \bar{\eta}^{-2} u^{2}\right) \mathrm{d} \bar{\eta} \mathrm{d} x^{3}$,

which is very different from the one in the inflation paradigm. The canonical momentum conjugate to $u$ is defined as $\pi=$ $\partial \mathscr{L} / \partial u^{\prime}=u^{\prime}$.

In the quantization process, the field variable $u$ and its canonical momentum $\pi$ become operators $\hat{u}$ and $\hat{\pi}$, respectively. The operator $\hat{u}$ obeys the equation

$\hat{u}^{\prime \prime}-\left(m \bar{\eta}^{-\ell} \Delta+n \bar{\eta}^{-2}\right) \hat{u}=0$,

which is the same as (38). In general, the solution of the above equation can be given by

$\hat{u}(\bar{\eta}, \mathbf{x})=\int \frac{\mathrm{d}^{3} \mathbf{k}}{(2 \pi)^{3 / 2}} \frac{1}{\sqrt{2}}\left(u_{\mathbf{k}}^{*}(\bar{\eta}) e^{i \mathbf{k} \mathbf{x}} \hat{a}_{\mathbf{k}}^{-}+u_{\mathbf{k}}(\bar{\eta}) e^{-i \mathbf{k} \mathbf{x}} \hat{a}_{\mathbf{k}}^{+}\right)$,

where $u_{\mathbf{k}}(\bar{\eta})$ satisfies

$u_{\mathbf{k}}^{\prime \prime}+\left(m \bar{\eta}^{-\ell} \mathbf{k}^{2}-n \bar{\eta}^{-2}\right) u_{\mathbf{k}}=0$,

and the bosonic commutation relations are given for the creation and annihilation operators as follows:

$\left[\hat{a}_{\mathbf{k}}^{-}, \hat{a}_{\mathbf{k}^{\prime}}^{-}\right]=\left[\hat{a}_{\mathbf{k}}^{+}, \hat{a}_{\mathbf{k}^{\prime}}^{+}\right]=0, \quad\left[\hat{a}_{\mathbf{k}}^{-}, \hat{a}_{\mathbf{k}^{\prime}}^{+}\right]=\delta^{(3)}\left(\mathbf{k}-\mathbf{k}^{\prime}\right)$.

The vacuum $|0\rangle$ is defined as the state which is annihilated by $a_{\mathbf{k}}^{-}$, i.e., $a_{\mathbf{k}}^{-}|0\rangle=0$. One requires $u_{\mathbf{k}}(\eta)$ to satisfy the normalization condition

$u_{\mathbf{k}}^{\prime} u_{\mathbf{k}}^{*}-u_{\mathbf{k}} u_{\mathbf{k}}^{* \prime}=2 i$,

of which the left-hand-side term is the Wronskian of (38). At any given time, thus, the operators $\hat{u}$ and $\hat{\pi}$ satisfy the commutation relations, i.e.,

$[\hat{u}(\bar{\eta}, \mathbf{x}), \hat{u}(\bar{\eta}, \mathbf{y})]=[\hat{\pi}(\bar{\eta}, \mathbf{x}), \hat{\pi}(\bar{\eta}, \mathbf{y})]=0$,

$[\hat{u}(\bar{\eta}, \mathbf{x}), \hat{\pi}(\bar{\eta}, \mathbf{y})]=i \delta^{(3)}(\mathbf{x}-\mathbf{y})$.

Equation (38) has two independent solutions which are represented in terms of the Bessel functions, namely,

$u_{\mathbf{k}}^{(1)}(\bar{\eta})=\bar{\eta}^{\frac{1}{2}} J_{\frac{\sqrt{1+4 n}}{2-\ell}}\left(\frac{2 \sqrt{m} k}{\ell-2} \bar{\eta}^{\frac{2-\ell}{2}}\right)$,

$u_{\mathbf{k}}^{(2)}(\bar{\eta})=\bar{\eta}^{\frac{1}{2}} Y_{\frac{\sqrt{1+4 h}}{2-\ell}}\left(\frac{2 \sqrt{m} k}{\ell-2} \bar{\eta}^{\frac{2-\ell}{2}}\right)$,

where we denote $k^{2}=\mathbf{k}^{2}$. Thus, its general solution can be expressed as $u_{\mathbf{k}}(\bar{\eta})=c_{1} u_{\mathbf{k}}^{(1)}(\bar{\eta})+c_{2} u_{\mathbf{k}}^{(2)}(\bar{\eta})$.

Note the Abel identity $J_{\alpha}(x) \frac{\mathrm{d} Y_{\alpha}(x)}{\mathrm{d} x}-\frac{\mathrm{d} J_{\alpha}(x)}{\mathrm{d} x} Y_{\alpha}(x)=\frac{2}{\pi x}$ for the Bessel functions. We could formally give the coefficients $c_{1}$ and $c_{2}$ as follows:

$c_{1}=\sqrt{\frac{\pi \eta_{e}}{2-\ell}}, \quad c_{2}=-i c_{1}$.

Here we have disregarded a common complex-number factor, which is unitary. In the UV regime, if $\lambda=0$, the above solution coincides with the standard formula $u_{\mathbf{k}} \sim$ $\frac{1}{\sqrt{c_{s} k}} e^{-i c_{s} k \eta}$ in the Minkowski spacetime. The reason is that $J_{\alpha}(x)-i Y_{\alpha}(x)$ has the asymptotic expression which is proportional to $\sqrt{\frac{2}{\pi x}} e^{-i x}$ when $x \gg 0$. By substituting (50) into (49), we obtain the general representation for $u_{\mathbf{k}}(\bar{\eta})$.

\section{Primordial power spectrum}

We are particularly interested in the long-wavelength perturbations. At the initial moment, these modes are deep inside the Hubble horizon because of the large value for the effective speed of light. With the decrease of the temperature, the effective speed of light decreases rapidly. Thus, these modes would exit from the Hubble horizon. After the dominating era of the gravity's rainbow effects, they reentered the Hubble horizon with the expansion of the universe. In the IR regime, the Bessel function with $\alpha>0$ has the asymptotic expressions $J_{\alpha}(x) \rightarrow \frac{1}{\Gamma(\alpha+1)}\left(\frac{x}{2}\right)^{\alpha}$ and $Y_{\alpha}(x) \rightarrow-\frac{\Gamma(\alpha)}{\pi}\left(\frac{2}{x}\right)^{\alpha}$. Thus, the term in $Y_{\alpha}(x)$ will dominate for the long-wavelength perturbations. Therefore, the power spectrum for the gravitational potential $\Phi$ is given by

$$
\begin{aligned}
P_{\Phi}(k) & =\frac{k^{3}}{2 \pi^{2}}\left\langle\Phi_{\mathbf{k}}^{*} \Phi_{\mathbf{k}}\right\rangle \propto k^{3} \eta^{-2 q}\left\langle u_{\mathbf{k}}^{*} u_{\mathbf{k}}\right\rangle \\
& \propto k^{3-2 \frac{\sqrt{1+4 n}}{\ell-2}} \eta^{1-2 q-\sqrt{1+4 n}} \\
& \propto k^{\frac{4(\lambda-3)}{3 \lambda-2}}
\end{aligned}
$$

where we have used $u \propto(-\eta)^{q} \Phi$, and in the last step the relation $k=\mathscr{H} / c \propto a^{\frac{3 \lambda-2}{2}} \propto(-\eta)^{\frac{3 \lambda-2}{2-\lambda}}$ for the horizon-crossing modes. A more detailed calculation can give the amplitude for the above power spectrum of gravitational potential. In fact, the amplitude $A_{\Phi}$ is given by $\frac{3 \Gamma^{2}(\alpha)}{2 \pi^{3}(\lambda+1)}\left(\frac{\lambda-2}{2}\right)^{\frac{-2(\lambda+4)}{3 \lambda-2}} k_{\text {pivot }}^{\frac{4(\lambda-3)}{3 \lambda-2}} M^{\frac{2(\lambda+4)}{3 \lambda-2}}\left(\frac{3(3 \lambda-2)}{\lambda+1}\right)^{2 \alpha-1}$, where

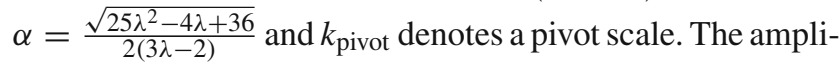
tude $A_{\Phi}$ is proportional to $M^{2}$ for the scale-invariant spectrum. To roughly estimate the magnitude of quantities for the energy scale $M$, one could approximate the amplitude as $A_{\Phi} \simeq M^{\frac{2(\lambda+4)}{3 \lambda-2}}$. Under the limit $\lambda=0$, the universe becomes radiation-dominated in our model. The cosmological perturbations will be mainly determined by the fluctuations of radi- 
ations, whose amplitude $\zeta$ is scaled as $1 / \sqrt{V}$. Here $V$ denotes a volume related to Hubble horizon. On the other hand, the volume $V$ is proportional to $k^{-3}$ for the horizon-crossing modes. Here $k$ denotes the wavenumber of a horizon-crossing mode. As a convention, the power spectrum of cosmological perturbations is usually defined as $P(k)=\frac{k^{3}}{2 \pi^{2}}|\zeta|^{2}$ in cosmology. Therefore, we can obtain the power spectrum, which is given by $P(k) \sim k^{3}(1 / \sqrt{V})^{2} \sim k^{6}$ in the limit $\lambda=0$.

Once the power spectrum for the gravitational potential is obtained, we can immediately obtain the power spectrum for the comoving curvature perturbation. By using (31) and (34) and transforming to the momentum space, we can represent the comoving curvature perturbation (35) in terms of the gravitational potential $\Phi_{\mathbf{k}}$, namely,

$\mathscr{R}_{\mathbf{k}}=-\Phi_{\mathbf{k}}\left[1+\frac{2}{3(1+\omega)}\left(1+\frac{\Phi_{\mathbf{k}}^{\prime} / \Phi_{\mathbf{k}}}{a^{\prime} / a}\right)\right]$,

where we have used the Friedmann equation. The term in the square bracket is a constant in the above representation. We denote it by $A$. Thus, the power spectrum of $\mathscr{R}_{\mathbf{k}}$ could be given by $P_{\mathscr{R}}(k)=\left(A^{*} A\right) P_{\Phi}(k)$. Here we can calculate $A$ by using $\Phi \propto \eta^{-q} u$ and the asymptotic IR expression of $u_{\mathbf{k}}(\eta)$, and the result is $A=$ $\frac{1}{2(\lambda+4)}\left[\sqrt{25 \lambda^{2}-4 \lambda+36}-3(\lambda-2)\right]$. Once $\lambda$ is determined, one can calculate $A$ and then obtain the power spectrum for the scalar perturbations. Finally, thus, the power spectrum of scalar perturbations can be parameterized as

$P_{\mathscr{R}}(k)=A_{\mathscr{R}}\left(\frac{k}{k_{\text {pivot }}}\right)^{\frac{4(\lambda-3)}{3 \lambda-2}}$,

where the amplitude is given by $A_{\mathscr{R}}=|A|^{2} A_{\Phi}$ and $k_{\text {pivot }}$ denotes the pivot scale. The scale-invariant power spectrum is given by $\lambda=3$. This result is different from that of $\lambda=2 \mathrm{in}$ other work [15-18]. Actually, this issue can be demonstrated by the terms proportional to $\frac{\mathrm{d} \ln c}{\mathrm{~d} \ln a}$ in Eq. (33). These terms show that the effective speed of light can be decreased with the expansion of the universe. They lead to the equation of motion (42) for the scalar perturbations. In this equation, the term proportional to $u_{\mathbf{k}}$ is different from the one in other work. Thus, we get different results.

The astronomical observations can give certain constraints on the parameters of the gravity's rainbow scenario. In this paper, we use the Planck TT [24] and WMAP polarization [25] datasets to set constraints on the rainbow index $\lambda$ and the energy scale $M$ for the gravity's rainbow effects via the CosmoMC [28]. The constraints on $\lambda$ and $M$ are given by

$\lambda=2.931 \pm 0.012, \quad \ln \left(10^{5} M\right)=-0.401_{-0.451}^{+0.457}$,

at the $68 \% \mathrm{CL}$, respectively. Here the pivot scale is chosen as $k_{\text {pivot }}=0.05 \mathrm{Mpc}^{-1}$. Thus, the gravity's rainbow effects

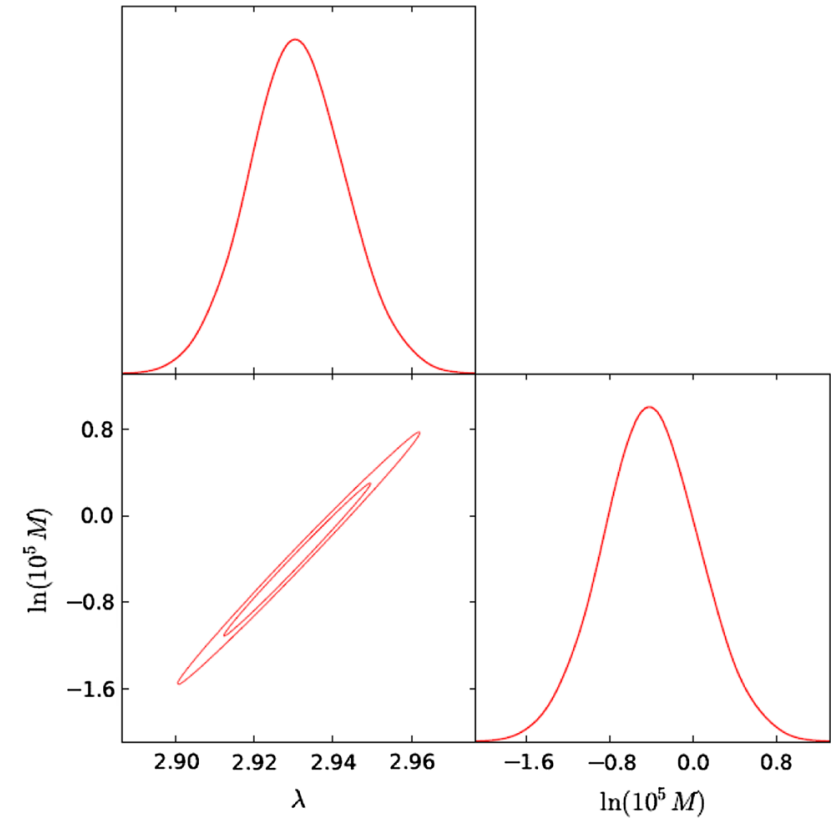

Fig. 1 The marginalized contour plot and the likelihood distributions of the rainbow index $\lambda$ and energy scale $\ln \left(10^{5} M\right)$ in the gravity's rainbow scenario

would become significant above the energy scale $\sim 10^{14} \mathrm{GeV}$. In addition, the marginalized contour plot and the likelihood distributions of $\lambda$ and $\ln \left(10^{5} M\right)$ are illustrated in Fig. 1.

\section{Conclusions and discussions}

In this paper, we have proposed that the gravity's rainbow scenario could be an alternative for the inflation paradigm of the very early universe. The rainbow function in the metric induces an effective speed of light which depends on the energy of moving particles. We studied the thermodynamics of the system of ultra-relativistic particles with the modified dispersion relation induced by the quantum gravity effects. Then the evolution of the very early universe is determined by the modified Friedmann equation, of which the solution was resolved. Furthermore, we have studied the cosmological linear perturbations and their quantization. The equations for the cosmological perturbations have been derived and the issue of gauge choices was discussed. In the longitudinal gauge, we studied the quantum cosmological perturbations, and then obtained the power spectrum for the primordial comoving curvature perturbations. Furthermore, we set constraints on the rainbow index $\lambda$ and the energy scale $M$ of the gravity's rainbow scenario by jointly analyzing the Planck TT and WMAP polarization datasets. Note that the nearly scale-invariant power spectrum for the scalar perturbations required $\lambda \simeq 3$, which satisfies the condition $2 / 3<\lambda<4$ to account for the flatness and horizon problems.

Though it shed light on the study of the very early universe, our phenomenological scenario still suffers certain puzzling 
issues. First, the large value for the rainbow function is related with a very high energy scale at the start time of the rainbow universe. At such a high energy scale, the quantum gravity effects are unclear. Second, the Einstein equation should be modified to account for the quantum gravity effects. However, we used the modified Einstein's equation with the speed of light replaced by the effective speed of light. Even though this equation could be reduced back to the conventional one in general relativity, it should be demonstrated by a consistent theory of quantum gravity in principle. Third, the rainbow metric belongs to the Riemann-Finsler geometry [29], whose dynamics has not been clearly studied so far. In conclusion, one still requires a complete and consistent theory of quantum gravity to study the very early universe in the future. Even though there were problems for the gravity's rainbow scenario, our studies still show some interesting results for the research of the very early universe.

Acknowledgments We acknowledge the use of Planck Legacy Archive, ITP, and Lenovo Shenteng 7000 supercomputer in the Supercomputing Center of Chinese Academy of Science for providing computing resources. We are grateful to Prof. Qing-Guo Huang, Xin Li, and Yi Ling for useful discussions. The author S.W. thanks Dr. Tian-Fu $\mathrm{Fu}$, Yue Huang, and Yu-Hang Xing for discussing some details in this paper. He also thanks for the hospitality at the Institute of Astronomy and Space Science in the Sun Yat-Sen University. The author Z.C. is funded by the Natural Science Fund of China (NSFC) under Grant No. 11375203 , and the author S.W. is supported by the project of Knowledge Innovation Program of Chinese Academy of Sciences and grants from NSFC (Grant Nos. 11322545, 11335012).

Open Access This article is distributed under the terms of the Creative Commons Attribution 4.0 International License (http://creativecomm ons.org/licenses/by/4.0/), which permits unrestricted use, distribution, and reproduction in any medium, provided you give appropriate credit to the original author(s) and the source, provide a link to the Creative Commons license, and indicate if changes were made. Funded by SCOAP ${ }^{3}$.

\section{References}

1. A.H. Guth, The inflationary universe: a possible solution to the horizon and flatness problems. Phys. Rev. D 23, 347 (1981)

2. A.D. Linde, A new inflationary universe scenario: a possible solution of the horizon, flatness, homogeneity, isotropy and primordial monopole problems. Phys. Lett. B 108, 389 (1982)

3. A. Albrecht, P.J. Steinhardt, Cosmology for grand unified theories with radiatively induced symmetry breaking. Phys. Rev. Lett. 48, 1220 (1982)

4. V.F. Mukhanov, H.A. Feldman, R.H. Brandenberger, Theory of cosmological perturbations. Part 1. Classical perturbations. Part 2. Quantum theory of perturbations. Part 3. Extensions. Phys. Rep. 215, 203 (1992)

5. P. Penrose, Difficulties with inflationary cosmology. Ann. N. Y. Acad. Sci. 271, 249 (1989)

6. A. Borde, A.H. Guth, A. Vilenkin, Inflationary space-times are incompletein past directions. Phys. Rev. Lett. 90, 151301 (2003). arXiv:gr-qc/0110012
7. S.M. Carroll, J. Chen, Does inflation provide natural initial conditions for the universe? Gen. Relativ. Gravit. 37, 1671 (2005). [Int. J. Mod. Phys. D 14, 2335 (2005)]. arXiv:gr-qc/0505037

8. R.H. Brandenberger, J. Martin, Trans-Planckian issues for inflationary cosmology. Class. Quantum Gravity 30, 113001 (2013). arXiv:1211.6753 [astro-ph.CO]

9. J. Magueijo, L. Smolin, Gravity's rainbow. Class. Quantum Gravity 21, 1725 (2004). arXiv:gr-qc/0305055

10. S. Weinfurtner, P. Jain, M. Visser, C.W. Gardiner, Cosmological particle production in emergent rainbow spacetimes. Class. Quantum Gravity 26, 065012 (2009). arXiv:0801.2673 [gr-qc]

11. Y. Ling, Q. Wu, The big bounce in rainbow universe. Phys. Lett. B 687, 103 (2010). arXiv:0811.2615 [gr-qc]

12. C. Corda, Primordial inflation from gravity's rainbow. AIP Conf. Proc. 1281, 847 (2010). arXiv:1007.4087 [gr-qc]

13. R. Garattini, M. Sakellariadou, Does gravity's rainbow induce inflation without an inflaton? Phys. Rev. D 90, 043521 (2014). arXiv: 1212.4987 [gr-qc]

14. B. Majumder, Singularity free rainbow universe. Int. J. Mod. Phys. D 22, 1342021 (2013). arXiv:1305.3709 [gr-qc]

15. G. Amelino-Camelia, M. Arzano, G. Gubitosi, J. Magueijo, Rainbow gravity and scale-invariant fluctuations. Phys. Rev. D 88(4), 041303 (2013). arXiv: 1307.0745 [gr-qc]

16. G. Amelino-Camelia, M. Arzano, G. Gubitosi, J. Magueijo, Dimensional reduction in the sky. Phys. Rev. D 87(12), 123532 (2013). arXiv:1305.3153 [gr-qc]

17. S. Mukohyama, Scale-invariant cosmological perturbations from Horava-Lifshitz gravity without inflation. JCAP 0906, 001 (2009). arXiv:0904.2190 [hep-th]

18. J. Magueijo, DSR as an explanation of cosmological structure. Class. Quantum Gravity 25, 202002 (2008). arXiv:0807.1854 [grqc]

19. A. Awad, A.F. Ali, B. Majumder, Nonsingular rainbow universes. JCAP 1310, 052 (2013). arXiv:1308.4343 [gr-qc]

20. J.D. Barrow, J. Magueijo, Intermediate inflation from rainbow gravity. Phys. Rev. D 88(10), 103525 (2013). arXiv:1310.2072 [astroph.CO]

21. J.W. Moffat, Superluminary universe: a possible solution to the initial value problem in cosmology. Int. J. Mod. Phys. D 2, 351 (1993). arXiv:gr-qc/9211020

22. A. Albrecht, J. Magueijo, A time varying speed of light as a solution to cosmological puzzles. Phys. Rev. D 59, 043516 (1999). arXiv:astro-ph/9811018

23. J.D. Barrow, J. Magueijo, Solving the flatness and quasiflatness problems in Brans-Dicke cosmologies with a varying light speed. Class. Quantum Gravity 16, 1435 (1999). arXiv:astro-ph/9901049

24. P.A.R. Ade et al. [Planck Collaboration], Planck 2013 results. XVI. Cosmological parameters. Astron. Astrophys. A 571, 16 (2014). arXiv:1303.5076 [astro-ph.CO]

25. G. Hinshaw et al. [WMAP Collaboration], Nine-year Wilkinson microwave anisotropy probe (WMAP) observations: cosmological parameter results. Astrophys. J. Suppl. 208, 19 (2013). arXiv:1212.5226 [astro-ph.CO]

26. Y. Ling, Rainbow universe. JCAP 0708, 017 (2007). arXiv:gr-qc/0609129

27. V. Mukhanov, Physical Foundations of Cosmology (Univ. Press, Cambridge, 2005)

28. A. Lewis, S. Bridle, Cosmological parameters from CMB and other data: a Monte Carlo approach. Phys. Rev. D 66, 103511 (2002). arXiv:astro-ph/0205436

29. X. Mo, An Introduction to Finsler Geometry (World Scientific Publishing Co., Ptc. Ltd, Singapore, 2006) 